\title{
Total Domination Subdivision Number in Strong Product Graph
}

\author{
P. Jeyanthi ${ }^{1, *}$, G. Hemalatha ${ }^{2}$, B. Davvaz ${ }^{3}$ \\ ${ }^{1}$ Department of Mathematics, Govindammal Aditanar College for Women, Tiruchendur, Tamil Nadu, India \\ ${ }^{2}$ Department of mathematics, Shri Andal Alagar College of Engineering, Mamandur, Kancheepuram, Tamil Nadu, India \\ ${ }^{3}$ Department of Mathematics, Yazd University, Yazd, Iran \\ *Corresponding author: jeyajeyanthi@rediffmail.com
}

Received June 03, 2014; Revised July 25, 2014; Accepted July 28, 2014

\begin{abstract}
A set $D$ of vertices in a graph $\mathrm{G}(\mathrm{V}, \mathrm{E})$ is called a total dominating set if every vertex $\mathrm{v} \in \mathrm{V}$ is adjacent to an element of $D$. The domination subdivision number of a graph $G$ is the minimum number of edges that must be subdivided in order to increase the domination number of a graph. In this paper, we determine the total domination number for strong product graph and establish bounds on the total domination subdivision number for strong product graph.
\end{abstract}

Keywords: total dominating set, strong product graph, total domination number

Cite This Article: P. Jeyanthi, G. Hemalatha, and B. Davvaz, "Total Domination Subdivision Number in Strong Product Graph.” American Journal of Applied Mathematics and Statistics, vol. 2, no. 4 (2014): 216-219. doi: 10.12691/ajams-2-4-7.

\section{Introduction}

Let $G=(V, E)$ be a simple graph on the vertex set $V$. In a graph $G$, a set $D \subseteq V$ is a dominating set of $G$ if every vertex in $V-D$ is adjacent to some vertex in $D$. The domination number of a graph $G$ is the minimum size of a dominating set of vertices in $G$, denoted by $\gamma(G)$. A thorough study of fundamental domination appears in [2]. The concept of total domination in graphs was introduced by Cokayne, Dawes and Hedetemini [1]. A set of vertices in a graph $G(V, E)$ is called a total dominating set if every vertex $v \epsilon V$ is adjacent to an element of $S$. The total domination number of some cartesian products of two paths $P_{n}$ and $P_{m}$, are investigated in [8,9]. The values of $\gamma_{t}\left(P_{n, m}\right)$ for $n=2,3,4$ are determined in [8], and for $n=$ 5,6 are determined in [9].

Let $G$ and $H$ be the two graphs with the set of vertices $U=\left\{u_{1}, u_{2}, \ldots, u_{n}\right\}$ and $V=\left\{v_{1}, v_{2}, \ldots, v_{m}\right\}$ respectively. The strong product of $\mathrm{G}$ and $\mathrm{H}$ is the graph $G \otimes H$ formed by the vertices $V=\left\{\left(u_{i}, v_{j}\right): 1 \leq i \leq n, 1 \leq j \leq m\right\}$ and two vertices $\left(u_{i}, v_{j}\right)$ and $\left(u_{s}, v_{t}\right)$ are adjacent in $G \otimes H \quad$ if and only if $\left(u_{i}=u_{s} \operatorname{and}_{j} \operatorname{adjv}_{t}\right)$, $\left(u_{i} a d j u_{s}\right.$ and $\left.v_{j}=v_{t}\right)$ or $\left(u_{i} a d j u_{s}\right.$ and $\left.v_{j} a d j v_{t}\right)$. Domination number is rather difficult to construct graphs with large value of $\operatorname{Sd} \gamma(G), S d \gamma_{t}(G)$ and the first conjecture on this subject was that $\operatorname{Sd} \gamma(G) \leq 3$ for every $\mathrm{G}$ [10]. The concept of total domination subdivision number $S d \gamma_{t}(G)$ was due to Haynes et al [3]. Haynes et.al [4] studied the total domination subdivision number of graphs, for instance, they showed that $S d \gamma_{t}(G) \leq 3$ holds for a graph having three or more pair wise adjacent simplicial vertices. In [5] the authors proved the total domination subdivision number of trees. Constant upper bounds on the total domination number for several families of graphs were determined in [3]. Nasrin Soltankhah showed that for any $m, n \geq 3, \quad S d \gamma_{t}(G) \leq 3$ [7]. The behaviour of several graph parameters in product graphs has become an interesting topic of research [6]. G. Yero and J. A. Rodr'1guez-Vel'azquez [11] proved that for any $m, n \geq 2, \gamma\left(P_{m} \otimes P_{n}\right)=\left\{\left\lceil\frac{m}{3}\right\rceil\left\lceil\frac{n}{3}\right\rceil\right\}$. In this paper is to establish a bound of this type on $\operatorname{Sd} \gamma_{t}\left(P_{n} \otimes P_{m}\right)$.

\section{Main Result}

In this section, we first determine the value of the total domination number of $P_{m} \otimes P_{n}$ for $m \leq 4$. Since $P_{1} \otimes P_{n} \simeq P_{n}$, we have:

Proposition 2.1. For any $n \geq 2$, we have

$$
\gamma_{t}\left(P_{1} \otimes P_{n}\right)=\left\{\begin{array}{ccc}
\frac{n}{2} & \text { if } & n \equiv 0(\bmod 4) \\
\frac{n}{2}+1 & \text { if } & n \equiv 1,2,3(\bmod 4) .
\end{array}\right.
$$


Lemma 2.2. We have $\gamma_{t}\left(P_{1} \otimes P_{n}\right)=\left\{\begin{array}{l}2 \text { for } n=2,3,4 \\ 3 \text { for } n=5 .\end{array}\right.$

Proof: To obtain totally dominate the vertices $\left(u_{2}, v_{1}\right)$ and $\left(u_{2}, v_{2}\right)$, we need two vertices $\left(u_{1}, v_{1}\right)$ and $\left(u_{1}, v_{2}\right)$. Therefore, $\gamma_{t}\left(P_{2} \otimes P_{2}\right)=2$. Last column of $P_{2} \otimes P_{3}$ is totally dominated by $P_{2} \otimes P_{2}$. Hence, $\gamma_{t}\left(P_{2} \otimes P_{3}\right)=2$. Let us consider $P_{2} \otimes P_{3}$ as block $B$. The last three columns of $P_{2} \otimes P_{4}$ is block $B$. The first column of $P_{2} \otimes P_{4}$ can be totally dominated by $B$. Hence, $\gamma_{t}\left(P_{2} \otimes P_{4}\right)=2$. In $P_{2} \otimes P_{5}$, to totally dominate a vertex $\left(u_{1}, v_{4}\right)$, we need one vertex among $\left\{\left(u_{2}, v_{3}\right),\left(u_{2}, v_{4}\right),\left(u_{1}, v_{4}\right)\right\}$. Hence, $\gamma_{t}\left(P_{2} \otimes P_{5}\right)=3$. The first three columns of $P_{2} \otimes P_{5}$ is block $B$ and also the last column of $P_{2} \otimes P_{5}$ is totally dominated by the fourth column. This completes the proof.

Proposition 2.3. For any $n \geq 6$, we have

$$
\gamma_{t}\left(P_{2} \otimes P_{n}\right)=\left\{\begin{array}{ccc}
\frac{n}{2} & \text { if } & n \equiv 0(\bmod 4) \\
\frac{n+1}{2} & \text { if } & n \equiv 1,3(\bmod 4) \\
\frac{n+2}{2} & \text { if } & n \equiv 2(\bmod 4) .
\end{array}\right.
$$

Proof:

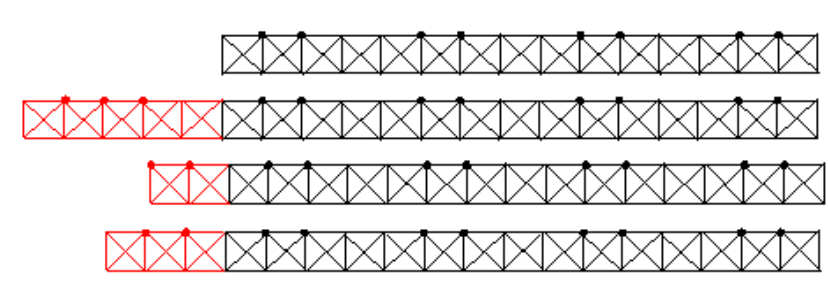

Figure 1. $P_{2} \otimes P_{n}$

Let $S$ be a total dominating set of $P_{2} \otimes P_{n}$. Since $\gamma_{t}\left(P_{2} \otimes P_{4}\right)=2$. Suppose that $C_{j}, C_{j+1}, C_{j+2}$ and $C_{j+3}$ are four consecutive columns of $P_{2} \otimes P_{n}$. To totally dominate the vertices $\left(u_{1}, v_{j+1}\right)$ and $\left(u_{1}, v_{j+2}\right)$, we need one vertex among $\left\{\begin{array}{l}\left(u_{1}, v_{j}\right),\left(u_{1}, v_{j+2}\right),\left(u_{2}, v_{j}\right), \\ \left(u_{2}, v_{j+1}\right),\left(u_{2}, v_{j+2}\right)\end{array}\right\}$ and one more vertex among $\left\{\begin{array}{l}\left(u_{1}, v_{j+1}\right),\left(u_{1}, v_{j+3}\right),\left(u_{2}, v_{j+1}\right), \\ \left(u_{2}, v_{j+2}\right),\left(u_{2}, v_{j+3}\right)\end{array}\right\}$. Now, to describe the total dominating set $S$, we consider block $B \simeq P_{2} \otimes P_{4} \quad$ and $\cap B=\left\{\left(u_{1}, v_{2}\right),\left(u_{1}, v_{3}\right)\right\}$. If $n \equiv 0(\bmod 4)$, then $P_{2} \otimes P_{n}$ can be partitioned with $\frac{n}{4}$ number of blocks $B$. If $n \equiv 1(\bmod 4)$, then $P_{2} \otimes P_{n}$ can be partitioned with $\frac{n-5}{4}$ number of blocks $B$, plus a block $B^{\prime} \simeq P_{2} \otimes P_{5}$ and $S \cap B^{\prime}=\left\{\left(u_{1}, v_{2}\right),\left(u_{1}, v_{3}\right),\left(u_{1}, v_{4}\right)\right\}$. If $n \equiv 2(\bmod 4)$, then $P_{2} \otimes P_{n}$ can be partitioned with $\frac{n-2}{4}$ number of blocks $B$, plus a block $B^{\prime} \simeq P_{2} \otimes P_{2}$ and $S \cap B^{\prime}=\left\{\left(u_{1}, v_{1}\right),\left(u_{1}, v_{2}\right)\right\}$. If $n \equiv 3(\bmod 4)$, then $P_{2} \otimes P_{n}$ can be partitioned with $\frac{n-3}{4}$ number of blocks $B$, plus a block $B^{\prime} \simeq P_{2} \otimes P_{3}$ and $S \cap B^{\prime}=\left\{\left(u_{1}, v_{1}\right),\left(u_{1}, v_{2}\right)\right\}$. This completes the proof.

Proposition 2.4. For $n \geq 3$, the total domination number of $P_{2} \otimes P_{n}$ and $P_{3} \otimes P_{n}$ are same.

Proof: Last two rows of $P_{3} \otimes P_{n}$ is considered as blocks $B \simeq P_{2} \otimes P_{n}$ and the first row of $P_{3} \otimes P_{n}$ is totally dominated by $B$, which completes the proof.

Observation 2.5. For $n \geq 1$, we have $P_{k} \otimes P_{n} \simeq P_{n} \otimes P_{k}$.

Proposition 2.6. For any $n \geq 4$, we have

Proof:
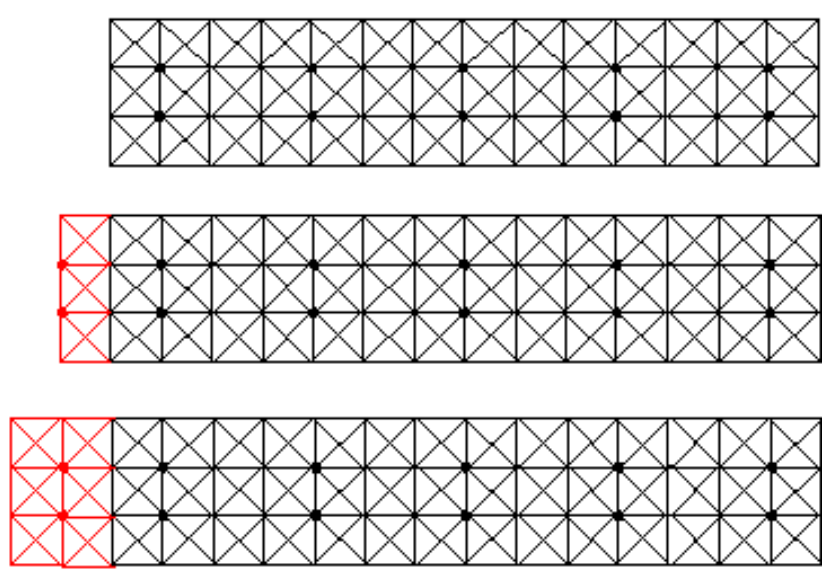

Figure 2. $P_{4} \otimes P_{n}$

Suppose that $S$ is a total dominating set of $P_{4} \otimes P_{n}$. Let us consider $P_{4} \otimes P_{3}$ as block. Since the total domination number of $P_{4} \otimes P_{3}$ is 2. We have $S \cap B=\left\{\left(u_{2}, v_{2}\right),\left(u_{3}, v_{2}\right)\right\}$. Let $C_{j}, C_{j+1}$ and $C_{j+2}$ be three consecutive columns of $P_{4} \otimes P_{n}$. To totally dominate the vertices $\left(u_{2}, v_{j+1}\right)$ and $\left(u_{3}, v_{j+1}\right)$, we need one vertex among $\left\{\begin{array}{l}\left(u_{1}, v_{j}\right),\left(u_{1}, v_{j+1}\right),\left(u_{1}, v_{j+2}\right), \\ \left(u_{2}, v_{j}\right),\left(u_{2}, v_{j+2}\right),\left(u_{3}, v_{j}\right), \\ \left(u_{3}, v_{j+1}\right),\left(u_{3}, v_{j+2}\right)\end{array}\right\}$ and one more vertex among

$$
\left\{\begin{array}{l}
\left(u_{2}, v_{j}\right),\left(u_{2}, v_{j+1}\right),\left(u_{2}, v_{j+2}\right),\left(u_{3}, v_{j}\right) \\
\left(u_{3}, v_{j+2}\right),\left(u_{4}, v_{j}\right),\left(u_{4}, v_{j+1}\right),\left(u_{4}, v_{j+2}\right)
\end{array}\right\} .
$$

Now, to describe our total dominating set $S$, we consider block $B \simeq P_{4} \otimes P_{3}$. If $n \equiv 0(\bmod 3)$, then $P_{4} \otimes P_{n}$ can be partitioned with $\frac{n}{3}$ number of blocks $B$. If $n \equiv 1(\bmod 3)$, then $P_{4} \otimes P_{n}$ can be partitioned with $\frac{n-1}{3}$ 
number of blocks $\mathrm{B}$, plus a block $B^{\prime} \simeq P_{4} \otimes P_{1}$ and $S \cap B^{\prime}=\left\{\left(u_{2}, v_{1}\right),\left(u_{3}, v_{1}\right)\right\} . \quad$ If $n \equiv 2(\bmod 3)$, then $P_{4} \otimes P_{n}$ can be partitioned with $\frac{n-2}{3}$ number of blocks $B$, plus a block $B^{\prime} \simeq P_{4} \otimes P_{2}$ and $S \cap B^{\prime}=\left\{\left(u_{2}, v_{1}\right),\left(u_{3}, v_{1}\right)\right\}$. This completes the proof.

Theorem 2.7. We have

$$
\gamma_{t}\left(P_{m} \otimes P_{n}\right)=\left\{\begin{array}{lll}
\left(\frac{m}{2}\right) \frac{n}{3} & \text { if } & m \equiv 0(\bmod 4) \\
\left(\frac{m}{2}+1\right) \frac{n}{3} & \text { if } & m \equiv 1,2,3(\bmod 4) .
\end{array}\right.
$$

\section{Proof:}

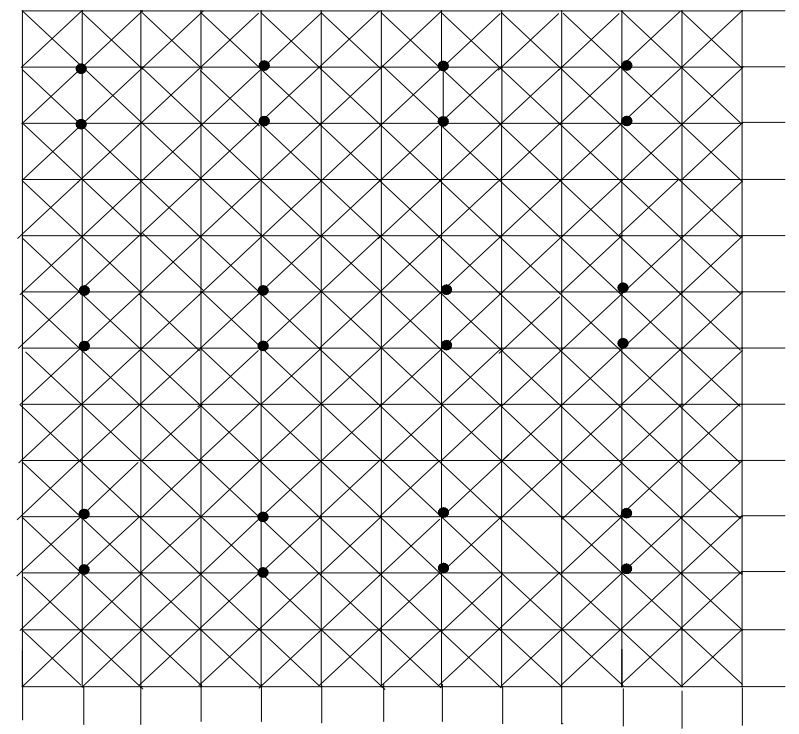

Figure 3. $P_{m} \otimes P_{n}$

Let $S$ be a total dominating set of $P_{m} \otimes P_{n}$. Since each column of $P_{m} \otimes P_{n}$ is isomorphic to $P_{m} \otimes P_{1}$. By Proposition 2.1 and Observation 2.5, we have

$$
\gamma_{t}\left(P_{m} \otimes P_{1}\right)=\left\{\begin{array}{lll}
\frac{m}{2} & \text { if } & m \equiv 0(\bmod 4) \\
\frac{m}{2}+1 & \text { if } & m \equiv 1,2,3(\bmod 4) .
\end{array}\right.
$$

Let us consider $P_{m} \otimes P_{1}$ as block. Now to describe our total dominating set $S$, we consider block $B \simeq P_{m} \otimes P_{1}$. If $m \equiv 0(\bmod 4)$, then $P_{m} \otimes P_{n}$ can be partitioned with $\left\lceil\frac{n}{3}\right\rceil$ number of blocks B. By Proposition 2.1 and Observation 2.5, $\quad$ we $\quad$ obtain $\quad \gamma_{t}\left(P_{m} \otimes P_{n}\right)=\left(\frac{m}{2}\right)\left\lceil\frac{n}{3}\right\rceil . \quad$ If $m \equiv 1,2,3,(\bmod 4)$, then $P_{m} \otimes P_{n}$ can be partitioned with $\left\lceil\frac{n}{3}\right\rceil$ number of blocks $B$. By Proposition 2.1 and Observation 2.5, we obtain $\gamma_{t}\left(P_{m} \otimes P_{n}\right)=\left(\left\lfloor\frac{m}{2}\right\rfloor+1\right)\left\lceil\frac{n}{3}\right\rceil$. This completes the proof.

\section{Subdivision Number for the Strong Product Graph}

Proposition 2.8. For $P_{2} \otimes P_{2}$, we have $S d \gamma_{t}\left(P_{2} \otimes P_{2}\right)=2$.

Proof: Let $S$ be a total dominating set of $P_{2} \otimes P_{2}$ and $S=\left\{\left(u_{1}, v_{1}\right),\left(u_{1}, v_{2}\right)\right\}$. Let $\left(P_{2} \otimes P_{2}\right)^{\prime}$ be obtain from $P_{2} \otimes P_{2}$ by subdividing an edge $\left(u_{1}, v_{1}\right)\left(u_{2}, v_{1}\right)$ and adding new vertex called $x$. Now, there is no change in total domination number, i.e, $\gamma_{t}\left(P_{2} \otimes P_{2}\right)^{\prime}=\gamma_{t r}\left(P_{2} \otimes P_{2}\right)$.

Let $\left(P_{2} \otimes P_{2}\right)$ " be obtain from $P_{2} \otimes P$ by subdividing the edges $\left(u_{1}, v_{1}\right)\left(u_{2}, v_{1}\right),\left(u_{2}, v_{1}\right)\left(u_{1}, v_{2}\right)$ and adding new vertices respectively called $x$ and $y$. So, we need three vertices for totally domination. Therefore, $S "=\left\{\left(u_{1}, v_{1}\right),\left(u_{1}, v_{2}\right),\left(u_{2}, v_{2}\right)\right\}$.

Thus, $\gamma_{t}\left(P_{2} \otimes P_{2}\right)^{\prime \prime}=3$. By Lemma.2.2, we obtain that the total domination number of $\left(P_{2} \otimes P_{2}\right)$ "' is greater than the total domination number of $P_{2} \otimes P_{2}$. This completes the proof.

Proposition 2.9. For $B \simeq P_{2} \otimes P_{2}$, we have $S \cap B=\left\{\left(u_{1}, v_{1}\right),\left(u_{1}, v_{2}\right)\right\}$.

Proof: To describe our total dominating set $S$, we consider block $B \simeq P_{2} \otimes P_{2}$ and $S \cap B=\left\{\left(u_{1}, v_{1}\right),\left(u_{1}, v_{2}\right)\right\}$. Since $S d \gamma_{t}\left(P_{2} \otimes P_{2}\right)=2$. Thus, we have $S d \gamma_{t}\left(P_{2} \otimes P_{3}\right)=2$.

Proposition 2.10. For $P_{2} \otimes P_{4}$, we have $S d \gamma_{t}\left(P_{2} \otimes P_{4}\right)=1$.

Proof: Let $S$ be a total dominating set of $P_{2} \otimes P_{4}$ and $S=\left\{\left(u_{1}, v_{2}\right),\left(u_{1}, v_{3}\right)\right\}$. Let $\left(P_{2} \otimes P_{4}\right)^{\prime}$ be obtain from $P_{2} \otimes P_{4}$ by subdividing an edge $\left(u_{2}, v_{1}\right)\left(u_{1}, v_{2}\right)$ and adding new vertex called $x$. To totally dominate $\left(u_{2}, v_{1}\right)$, we need one vertex among $\left\{x,\left(u_{1}, v_{1}\right),\left(u_{2}, v_{2}\right)\right\}$. Therefore, $\quad S^{\prime}=\left\{\left(u_{1}, v_{1}\right),\left(u_{1}, v_{2}\right),\left(u_{1}, v_{3}\right)\right\}$. Thus, $\gamma_{t}\left(P_{2} \otimes P_{4}\right)^{\prime}=3$.

By Lemma 2.2, we obtain that the total domination number of $\left(P_{2} \otimes P_{4}\right)^{\prime}$ is greater than the total domination number of $P_{2} \otimes P_{4}$. This completes the proof.

Proposition 2.11. For $P_{2} \otimes P_{5}$, we have $S d \gamma_{t}\left(P_{2} \otimes P_{5}\right)=1$.

Proof: Let $S$ be a total dominating set of $P_{2} \otimes P_{5}$ and $S=\left\{\left(u_{1}, v_{2}\right),\left(u_{1}, v_{3}\right),\left(u_{1}, v_{4}\right)\right\}$. Let $\left(P_{2} \otimes P_{5}\right)^{\prime}$ be obtain from $P_{2} \otimes P_{5}$ by subdividing an edge $\left(u_{2}, v_{1}\right)\left(u_{1}, v_{2}\right)$ and adding new vertex called $x$. To totally dominate $\left(u_{2}, v_{1}\right)$, we need one vertex among $\left\{x,\left(u_{1}, v_{1}\right),\left(u_{2}, v_{2}\right)\right\}$. Therefore, $S^{\prime}=\left\{\left(u_{1}, v_{1}\right),\left(u_{1}, v_{2}\right),\left(u_{1}, v_{3}\right),\left(u_{1}, v_{4}\right)\right\}$.

Thus, $\gamma_{t}\left(P_{2} \otimes P_{5}\right)^{\prime}=4$. By Lemma 2.2, we obtain that the total 
domination number of $\left(P_{2} \otimes P_{5}\right)$ ' is greater than the total domination number of $P_{2} \otimes P_{5}$. This completes the proof.

Theorem 2.8. For $n \geq 4$, we have $S d \gamma_{t}\left(P_{2} \otimes P_{n}\right)=1$.

Proof: To describe our total dominating set $S$, we consider block $B \simeq P_{2} \otimes P_{4}$ and $S \cap B=\left\{\left(u_{1}, v_{2}\right),\left(u_{1}, v_{3}\right)\right\}$. Since $S d \gamma_{t}\left(P_{2} \otimes P_{4}\right)=1$ and by Proposition 2.3, we have $S d \gamma_{t}\left(P_{2} \otimes P_{n}\right)=1$.

Theorem 2.9. For $n \geq 3$, subdivision number of $P_{2} \otimes P_{n}$ and $P_{3} \otimes P_{n}$ are same.

Proof: Last two rows of $P_{3} \otimes P_{n}$ is considered as blocks $B \simeq P_{2} \otimes P_{n}$ and the first row of $P_{3} \otimes P_{n}$ is totally dominated by $\mathrm{B}$, which completes the proof.

Theorem 2.10. For $n \geq 4$, we have $S d \gamma_{t}\left(P_{4} \otimes P_{n}\right)=1$.

Proof: To describe our total dominating set $S$, we consider block $B \simeq P_{4} \otimes P_{3}$ and $S \cap B=\left\{\left(u_{2}, v_{2}\right),\left(u_{3}, v_{2}\right)\right\}$. By Theorem 2.9, we have $\operatorname{Sd} \gamma_{t}\left(P_{4} \otimes P_{3}\right)=1$. Thus, $S d \gamma_{t}\left(P_{4} \otimes P_{n}\right)=1$.

Theorem 2.11. For $n \geq 4$, we have $S d \gamma_{t}\left(P_{m} \otimes P_{n}\right)=1$.

Proof: To describe our total dominating set $S$, we consider block $B \simeq P_{4} \otimes P_{n}$. By Theorem 2.10, we have $S d \gamma_{t}\left(P_{4} \otimes P_{n}\right)=1$. Thus, $S d \gamma_{t}\left(P_{m} \otimes P_{n}\right)=1$.

\section{References}

[1] E.J. Cockayne, R.M. Dawes and S.T. Hedetniemi, Total dominations in graphs, Networks, 10 (1980), 211-219.

[2] T.W. Haynes, S. T. Hedetniemi and P. J. Slater, Fundamentals of Domination in Graphs, Marcel Dekker, New York, 1997.

[3] T.W. Haynes, S.T. Hedetniemi and L.C. van der Merwe, Total domination subdivision numbers, J. Combin. Math. Combin. Comput., 44 (2003), 115-128.

[4] W. Haynes and Michael A. Henning, Total domination subdivision numbers of graphs, Discussiones Mathematicae Graph Theory 24(2004), 457-467.

[5] Teresa W. Haynes, Michael A. Henning and Lora Hopkins, Total domination subdivision numbers of trees, Discrete Mathematics 286(2004), 195-202.

[6] W. Imrich, S. Klav`zar, D. F. Rall, Topics in Graph Theory. A. K. Peters Ltd., Wellesley, MA, 2008.

[7] N.Soltankhah, On total domination subdivision number of grid graphs, Int.J.Contemp.Math.Sciences, 5(49) (2010), 2419-2432.

[8] N. Soltankhah, Results on total domination and total restrained domination in grid graphs, International Mathematical Forum, 5(7) (2010), 319-332.

[9] S. Gravier, Total domination number of grid graphs, Discrete Applied Mathematics, 121 (2002) 119-128.

[10] S. Velammal, "Studies in Graph Theory", Ph.D. Thesis (Manonmaniam Sundaranar University, Tirunelveli, 1997.

[11] G. Yero, J. A. Rodr'1guez-Vel'azquez, Roman domination in Cartesian product graphs and Strong product graph, Discrete Math., 7(2013), 262-274. 